\title{
Evaluation of the Thermal Resistance in GaN-Diodes by means of Electro-Thermal Monte Carlo Simulations
}

\author{
S. García*, I. Íñiguez-de-la-Torre, Ó. García-Pérez, J. Mateos, T. González and S. Pérez \\ Departamento de Física Aplicada, Universidad de Salamanca, \\ Plaza de la Merced s/n, 37008 Salamanca, Spain \\ Phone Number: (+34) 923294436 Fax Number: (+34) 923294584 *e-mail: sergio_gs@usal.es
}

\begin{abstract}
In this paper we use an electro-thermal method [solver of the heat-flux equation coupled with an ensemble Monte Carlo (MC) simulator] to extract the value of the thermal resistance, $R_{t h}$, in diodes consisting in un-gated $\mathrm{Al}_{0.27} \mathrm{Ga}_{0.73} \mathrm{~N} / \mathrm{GaN}$ heterostructures. Different substrates (polycrystalline diamond - PCD, diamond, silicon and sapphire), and die dimensions will be analysed. When a temperatureindependent thermal conductivity is considered, the obtained values of $R_{t h}$ depend on the geometry and substrate material, and are constant with the dissipated power $\left(P_{\text {diss }}\right)$. When a temperaturedependent thermal conductivity is needed to correctly reproduce the thermal behaviour of the device, $R_{t h}$ exhibits a strong dependence on $P_{\text {diss. }}$.
\end{abstract}

Keywords - Electrothermal modeling, Monte Carlo (MC), thermal resistance, high-temperature, AlGaN/GaN

\section{INTRODUCTION AND MODELING APPROACH}

In spite of the strong potentiality of $\mathrm{GaN}$ and $\mathrm{AlGaN} / \mathrm{GaN}$ based devices for high-power and high-frequency applications, their utilization is limited by the elevated lattice temperatures reached inside them. This excessive overheating is the main hindrance to their reliability and use [1]. Therefore, the correct description of self-heating effects is essential for the accurate modelling of GaN-based devices. In order to take into account thermal effects different techniques can be employed, such as a simple thermal resistance method (TRM) [2], or more complex procedures which take into consideration the solution of the steady state heat-diffusion-equation (HDE) [3]-[4].

Our home-made, semi-classical Monte Carlo (MC) simulator has been proved to be a very efficient tool to investigate electron transport and optimize the static, dynamic and noise operation of semiconductor devices [5]-[6]. In this contribution, we have expanded our simulator to a full electrothermal model. Specifically, it has been coupled with (i) a thermal resistance method, and (ii) the solution of the HDE [3]-[4]. A sufficient number of iterations of the HDEMC solver must be carried out in order to reach the convergence of the static electro-thermal solution [7].

The TRM is typically employed to evaluate the thermal behaviour of devices due to their simplicity and lower time consumption with respect to another algorithms. The lattice temperature, $T_{\text {latt, }}$ is updated according to the formula $T_{\text {latt }}=300+P_{\text {diss }} \times R_{\text {th }}$ (being $P_{\text {diss }}$ the dissipated power). In this approach the value of $R_{t h}$ is usually extracted phenomenologically. As an interesting alternative, the HDEMC simulator allows us not only to analyse the thermal behaviour itself, but also to extract the value of this thermal resistance, as illustrated in this work. Therefore the HDE-MC simulator can be used as a tool to evaluate the $R_{t h}$ dependence on the geometry and thermal parameters of the device.

The geometry of the $\mathrm{Al}_{0.27} \mathrm{Ga}_{0.73} \mathrm{~N} / \mathrm{GaN}$ diode under analysis is shown in Fig.1. The distance between the contacts is $2 \mu \mathrm{m}$. The electronic and thermal simulations take place in two different domains, also sketched in Fig. 1. Piezoelectric scattering and those with phonons and dislocations are included in the model. To correctly simulate the heterolayer we incorporate the influence of spontaneous and piezoelectric surface polarization charges: $P=12.12 \times 10^{12} \mathrm{~cm}^{-2}$. In addition, a surface charge density $\sigma=-4.12 \times 10^{12} \mathrm{~cm}^{-2}$ is placed at the top of the AlGaN layer, which appears as a result of polarization charges partially compensated by charge trapped in surface states [7].

A Dirichlet boundary condition for the HDE is imposed at the bottom of the structure (a heat sink with a constant temperature $300 \mathrm{~K}$ ). To correctly model the interfaces between layers, we use the continuity condition:

$$
k_{1} \partial T /\left.\partial r_{n}\right|_{\gamma}=k_{2} \partial T /\left.\partial r_{n}\right|_{\gamma}
$$

where $\gamma$ is the interface between two layers with thermal conductivities $k_{1}$ and $k_{2}, r_{n}$ the normal position vector and $T$ the temperature. The values considered for the thermal conductivities are: $k_{s}=30 \mathrm{~W} /(\mathrm{K} \cdot \mathrm{m})[8], k_{s}=130 \mathrm{~W} /(\mathrm{K} \cdot \mathrm{m})$ [9] and $k_{s}=300 \mathrm{~W} /(\mathrm{K} \cdot \mathrm{m})$ [4] for $\mathrm{AlGaN}, \mathrm{GaN}$ and $\mathrm{Au}$, respectively at $300 \mathrm{~K}$. Temperature independent and

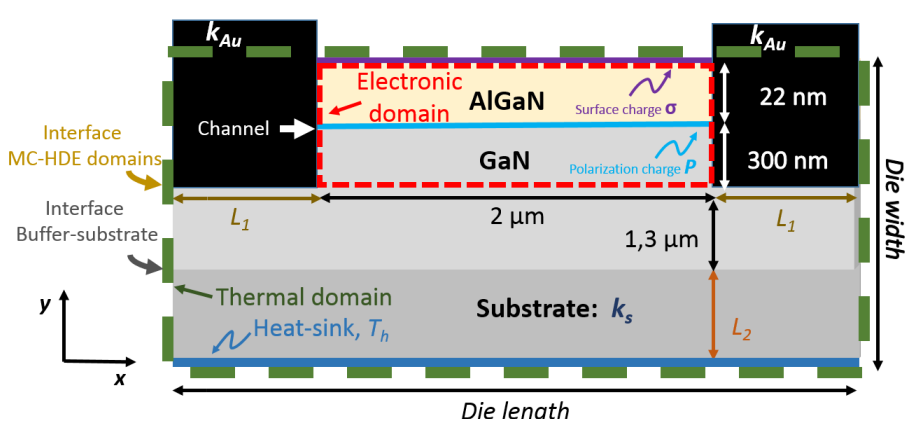

Fig. 1. Schematic structure of the $\mathrm{Al}_{0.27} \mathrm{Ga}_{0.73} \mathrm{~N} / \mathrm{GaN}$ diode under study. 
dependent thermal conductivities will be used in the simulations as it reported in next section.

\section{RESULTS AND DISCUSSION}

Firstly, considering temperature-independent thermal conductivities, we examine through the HDE-MC simulator the effect of varying (i) the thermal conductivity of the substrate $\left(k_{s}\right)$ and (ii) the semiconductor die dimensions $\left(L_{1}, L_{2}\right)$.

In Fig. 2 we plot, for devices grown on different substrates $\left(\mathrm{PCD}, k_{s}=2200 \mathrm{~W} /(\mathrm{K} \cdot \mathrm{m}) ;\right.$ diamond, $k_{s}=1000 \mathrm{~W} /(\mathrm{K} \cdot \mathrm{m}) ; \mathrm{Si}$, $k_{s}=156 \mathrm{~W} /(\mathrm{K} \cdot \mathrm{m})$ and sapphire, $\left.k_{s}=42 \mathrm{~W} /(\mathrm{K} \cdot \mathrm{m})\right)$, the typical $I-V$ curves, Fig. 2 (a), and the average temperature in the electronic domain $\left(T_{a v}\right) v s$. dissipated power, Fig. 2 (b). For the lower values of $k_{s}$, the self-heating becomes crucial: the substrate exhibits a significant influence on the current saturation level; note that for $V_{D S}=8 \mathrm{~V} T_{a v}=612 \mathrm{~K}$ for sapphire and $T_{a v}=438 \mathrm{~K}$ for $\mathrm{Si}$. In the case of materials with high $k_{s}$, although the PCD substrate has a thermal conductivity more than twice higher than the diamond substrate, the current level does not change significantly as a consequence of having similar $T_{a v},\left(355 \mathrm{~K}\right.$ and $365 \mathrm{~K}$ for $V_{D S}=8 \mathrm{~V}$, respectively). This reflects the fact that both substrates possess excellent thermal conductivities and can be considered as good heat-sinks.

Fig. 2 (b) allows us to calculate the values of $R_{t h}$ by fitting the $T_{a v}$ dependence $v s$. dissipated power obtained from HDE-MC simulations. The values of $R_{t h}$ are of the order of $10^{-2} \mathrm{~K} \cdot \mathrm{m} / \mathrm{W}$, well in the range of typical values for HEMTs [2] $\left(R_{t h}=38.2 \times 10^{-3} \mathrm{~K} \cdot \mathrm{m} / \mathrm{W}, \quad R_{t h}=14.1 \times 10^{-3} \mathrm{~K} \cdot \mathrm{m} / \mathrm{W}, \quad R_{t h}=4.6 \times 10^{-3}\right.$ $\mathrm{K} \cdot \mathrm{m} / \mathrm{W}$ and $R_{t h}=3.58 \times 10^{-3} \mathrm{~K} \cdot \mathrm{m} / \mathrm{W}$ for sapphire, $\mathrm{Si}$, diamond and PCD, respectively). We conclude that $R_{t h}$ is inversely proportional to $k_{s}$, inset of Fig 2 (b). Such estimated values for the thermal resistances will provide, within a TRM based model, the same $I-V$ curves than the simulations with the HDE-MC model [7]. Therefore, the feedback between both models can be used to speed-up the optimization of the device thermal properties, as the TRM approach is faster and require less memory resources. However, with the TRM just a global value of the lattice temperature is calculated, while with the electro-thermal model a local temperature map can be analysed, being possible the identification of hot spots inside the device in more complex devices like HEMTs.

In Fig. 3 we plot, for a $6 \mathrm{~V}$ bias, the lattice temperature in the thermal and electronic domains, for the PCD, Si, and sapphire substrates. A strong degradation of the device operation can take place with the sapphire substrate due to the very high temperatures reached as a consequence of the poor thermal conductivity [Fig. 3 (e), (f)] in comparison with $\mathrm{Si}$ [Fig. 3 (c), (d)] and PCD [Fig. 3 (a), (b)]. In the electronic domain, the average temperatures are $T_{a v}=336 \mathrm{~K}, T_{a v}=412 \mathrm{~K}$ and $T_{a v}=528 \mathrm{~K}$ (highest values of $345 \mathrm{~K}, 422 \mathrm{~K}$ and $535 \mathrm{~K}$ ) for the PCD, Si and sapphire respectively.
In Fig. 4 we represent the profiles of the increase of lattice temperature for the same bias at three different $y$-positions (channel, interface MC-HDE domains and interface buffersubstrate). In all cases the temperature peak is located well in the GaN two-dimensional electron gas (2DEG) of the diode. It is noteworthy that when the thermal conductivity is low the lattice temperature for the three layers is almost the same and constant (the sapphire material causes an enormous temperature rise), both in the electronic domain and thermal domain. In this particular case, and if the die length is too short, the contacts will be subjected to high temperatures (which could damage them) in comparison with the cases of diamond or PCD substrates. As was expected, the substrate exerts a strong influence over the self-heating of the device.

In Fig. 5 the dependence of $R_{t h}$ respect to die dimensions $L_{l}$ (bottom axis, $L_{2}=300 \mu \mathrm{m}$ ) and $L_{2}$ (top axis, $L_{l}=200 \mu \mathrm{m}$ ) is represented. In all cases the substrate employed is $\mathrm{Si}$. On the one hand, when $L_{l}$ increases above $250 \mu \mathrm{m}, R_{t h}$ remains almost constant, $13.3 \times 10^{-3} \mathrm{~K} \cdot \mathrm{m} / \mathrm{W}$. We have checked that values of $L_{1}$ lower than $100 \mu \mathrm{m}$ result in very high
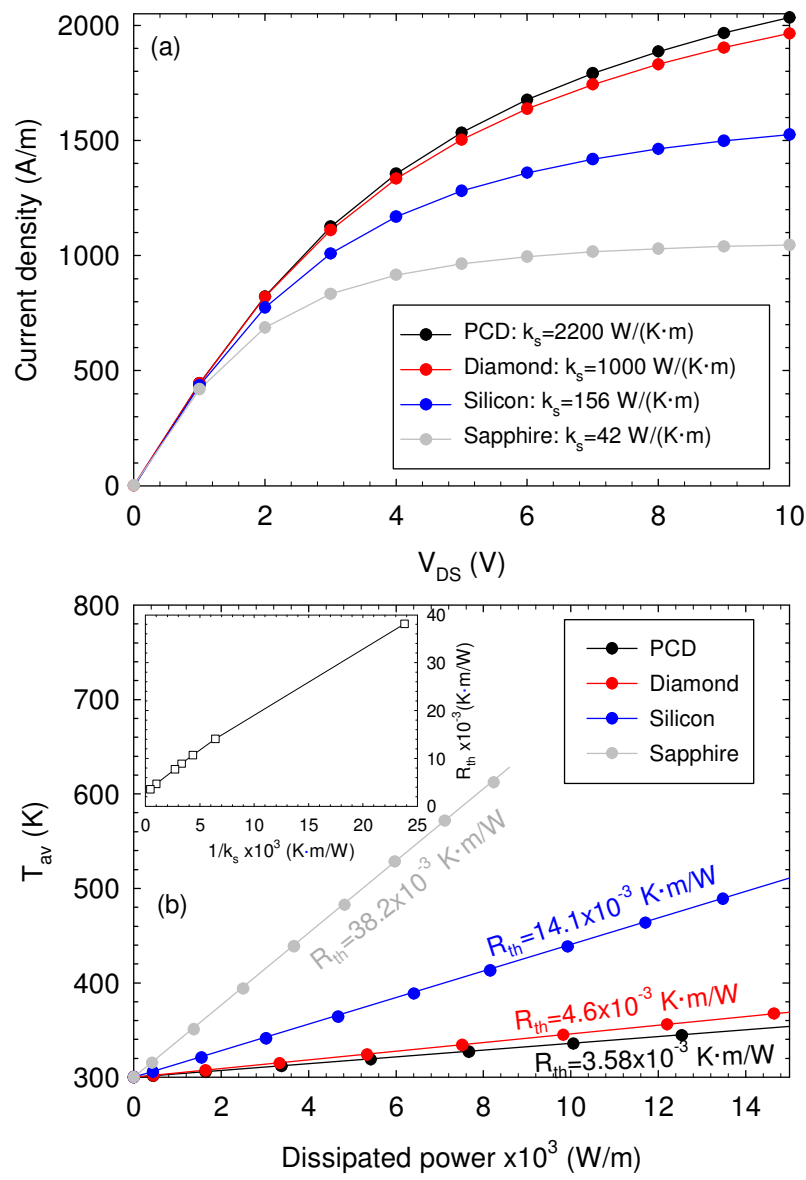

Fig. 2. (a) $I-V$ curves for different substrates: $\operatorname{PCD}\left[k_{\mathrm{s}}=2200 \mathrm{~W} /(\mathrm{K} \cdot \mathrm{m})\right]$, diamond $\left[k_{\mathrm{s}}=1000 \mathrm{~W} /(\mathrm{K} \cdot \mathrm{m})\right]$, silicon $\left[k_{\mathrm{s}}=156 \mathrm{~W} /(\mathrm{K} \cdot \mathrm{m})\right]$ and sapphire $\left[k_{\mathrm{s}}=42 \mathrm{~W} /(\mathrm{K} \cdot \mathrm{m})\right]$. (b) Analysis to evaluate the influence of $k_{s}$ in the simulations. Average temperature, $T_{a v}, v s$. dissipated power and linear fitting to extract the corresponding thermal resistance. The inset shows $R_{t h} v s .1 / k_{s}$. $L_{l}=200 \mu \mathrm{m}$ and $L_{2}=300 \mu \mathrm{m}$. 


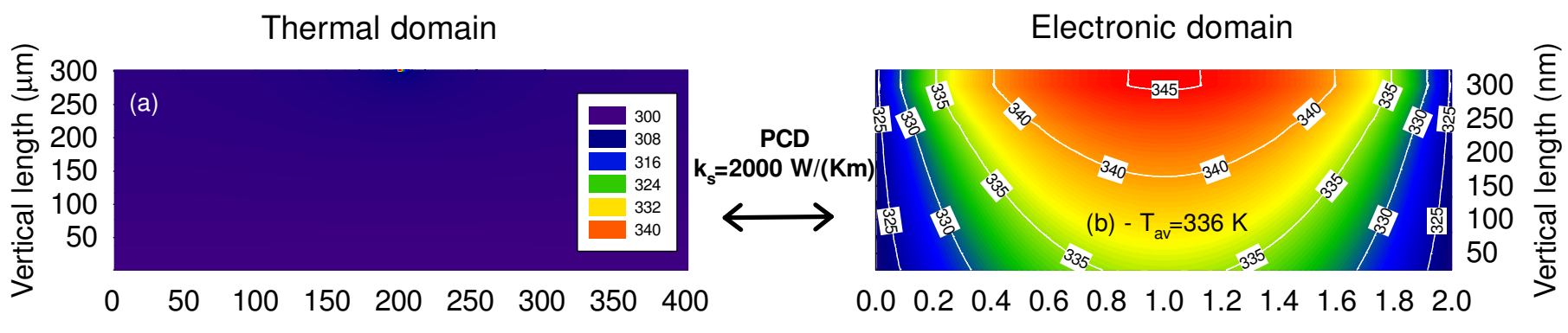

Horizontal length $(\mu \mathrm{m})$

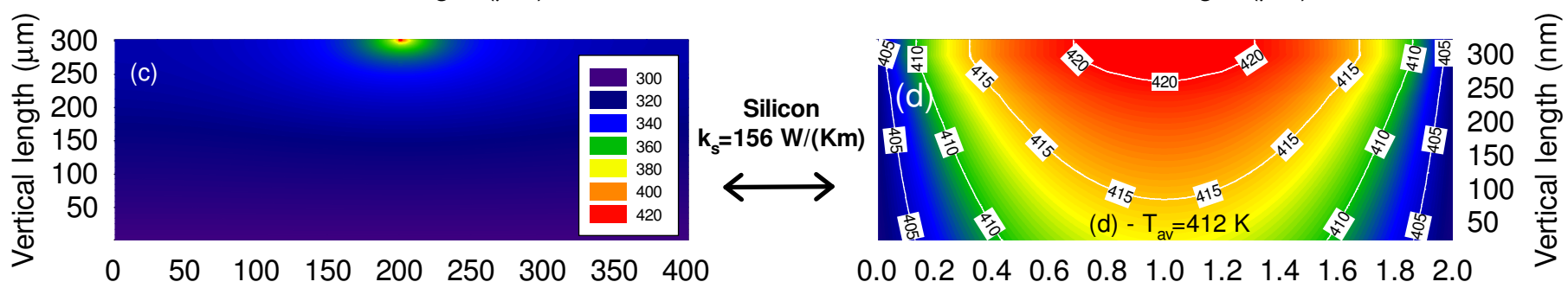

Horizontal length $(\mu \mathrm{m})$

Horizontal length $(\mu \mathrm{m})$

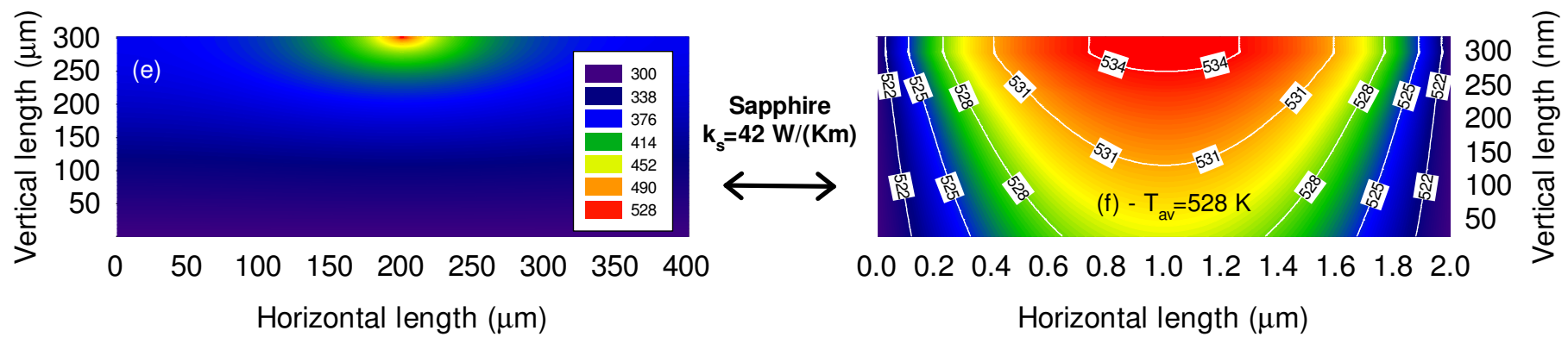

Fig. 3. Temperature distribution, for a bias of $6 \mathrm{~V}$, to evaluate the influence of $k_{s}: \mathrm{PCD}, 2200 \mathrm{~W} /(\mathrm{K} \cdot \mathrm{m})$; silicon, $156 \mathrm{~W} /(\mathrm{K} \cdot \mathrm{m})$ and sapphire, $42 \mathrm{~W} /(\mathrm{K} \cdot \mathrm{m})$ in the simulations. Thermal domain (a), (c) and (e), and electronic domain (b), (d) and (f).

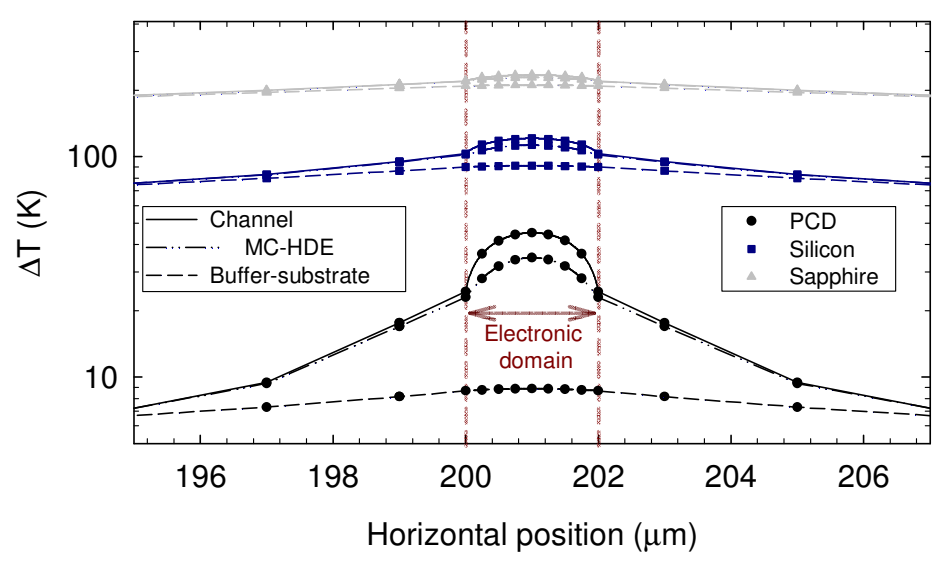

Fig. 4: Profile of the increase of lattice temperature $\left(\Delta T=T_{\text {latt }}-T_{h}\right)$ for three different $y$-positions (channel, interface MC-HDE domains and interface buffer-substrate, see Fig. 1) for a bias of $6 \mathrm{~V}$.

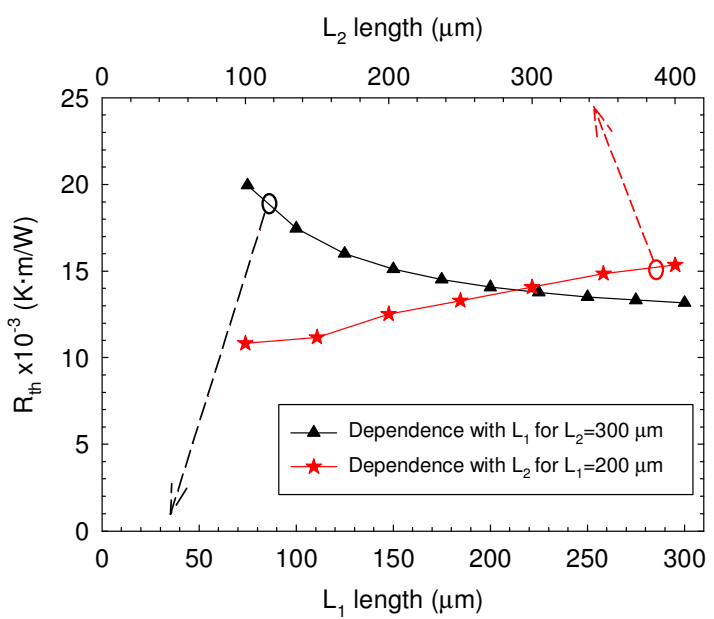

Fig. 5. $R_{t h} v s$. die dimensions (a) $L_{l}$ (bottom axis, $L_{2}=300 \mu \mathrm{m}$ ) and (b) $L_{2}$ (top axis, $\left.L_{l}=200 \mu \mathrm{m}\right)$. Both cases are for $k_{s}=156 \mathrm{~W} /(\mathrm{K} \cdot \mathrm{m})$. 
temperatures $>700 \mathrm{~K}$ at $V \sim 10 \mathrm{~V}$, providing values of $R_{t h}>20 \times 10^{-3} \mathrm{~K} \cdot \mathrm{m} / \mathrm{W}$. On the other hand, as $L_{2}$ is reduced, the thermal resistance is lower, as expected, and therefore heat flux generated by the phonons is dissipated more efficiently because the heat sink is closer to the electronic domain. $R_{t h}$ is in the range between $10-20 \times 10^{-3} \mathrm{~K} \cdot \mathrm{m} / \mathrm{W}$ in both cases.

As a second step, when the dissipated power is extremely high, it is appropriate to consider temperature-dependent thermal conductivities for each material. It is wellestablished that the thermal conductivity, $k_{i}$, depends on temperature as $A T^{-\alpha}$, where $A$ and $\alpha$ are characteristics parameters of each material. In order to fulfil the temperature continuity, and furthermore properly resolve the HDE when the Kirchhoff transformation is used, it is necessary to consider the same functional relation $T^{-\alpha}$ for all the involved $k_{i}$ [4]. For our particular study the value of $\alpha$ was set for all semiconductors to 1.3 [3]-[7]. It provides the best compromise for fitting the whole experimental ensemble of data in the temperature range of interest, and moreover it is the particular dependence of the $k_{i}$ corresponding to the most relevant layer $(\mathrm{Si})$ for the solution of the HDE, so that:

$$
k_{i}(T) \approx k_{i}^{300} \cdot\left(\frac{300 K}{T}\right)^{1.3} .
$$

In Fig. 6 we compare simulations using (i) a $k$-temperature independent model and (ii) a $k$-temperature dependent model, for Si substrate, $L_{2}=300 \mu \mathrm{m}$ and $L_{l}=200 \mu \mathrm{m}$. Up to $3 \mathrm{~V}$ both models provide same current level. For voltages greater than $3 \mathrm{~V}$, the reduction of the value of the thermal conductivities, according to Eq. 2, provokes an increase of temperature (see inset of Fig. 6), and obviously the current decreases.

Also it can be noted from the inset of Fig. 6 that the temperature-dependent thermal conductivity model does not give a linear response of the average temperature with respect to the dissipated power, being not possible to define a constant thermal resistance. As a consequence, a simple

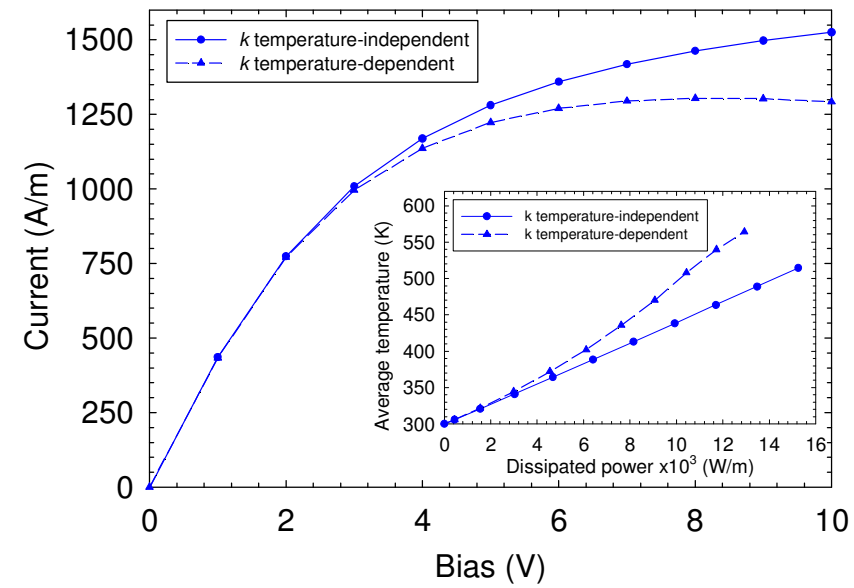

Fig. 6: (a) $I$ - $V$ curves obtained with both $k$-temperature independent and dependent models for Si substrate, $L_{2}=300 \mu \mathrm{m}$ and $L_{1}=200 \mu \mathrm{m}$. The inset shows the $T_{a v} v s$. dissipated power for the structure in both cases.
TRM cannot be applied and the HDE-MC scheme is required.

\section{CONCLUSIONS}

In this paper we have studied self-heating effects in an ungated $\mathrm{AlGaN} / \mathrm{GaN}$ diode. On the one hand, when a temperature-independent thermal conductivity is used in a HDE-MC simulator, it has been shown that by a linear fitting of $T_{\mathrm{av}}$ with respect to the intrinsic dissipated power it is possible to extract the value of $R_{t h}$. The parameter that has the strongest impact on $R_{t h}$ is $k_{\mathrm{s}}$, followed by $L_{1}$, and finally by $L_{2} . R_{t h}$ is extremely high for the sapphire substrate $\left(38.2 \times 10^{-3} \mathrm{~K} \cdot \mathrm{m} / \mathrm{W}\right)$, and remains almost constant $\left(\sim 13.3 \times 10^{-3} \mathrm{~K} \cdot \mathrm{m} / \mathrm{W}\right)$ from a length of $L_{l}=250 \mu \mathrm{m}$ when the Si-substrate is employed. On the other hand, when a temperature-dependent thermal conductivity is employed, $R_{t h}$ is function not only of the geometry and materials of the die, but also of the dissipated power. In this later case it would be necessary to carry out the HDE-MC simulations for a proper thermal study of the device.

\section{ACKNOWLEDGMENT}

This work has been partially supported by the Dirección General de Investigación Científica y Técnica (MINECO) through the Project TEC2013-41640-R and by the Consejería de Educación de la Junta de Castilla y León through the Project SA052U13.

\section{REFERENCES}

[1] R. Cuerdo et al., "High Temperature Microwave Performance of Submicron AlGaN/GaN HEMTs on SiC," IEEE Elec. Dev. Lett, vol. 30, pp. 808-810, January 2009.

[2] A. Asgari, M. Kalafi, and L. Faraone, "A quasi-two-dimensional charge transport model of $\mathrm{AlGaN} / \mathrm{GaN}$ high electron mobility transistors (HEMTs)," Physica E-Low-Dimensional Systems \& Nanostructures, vol. 28, pp. 491-499, July 2005.

[3] T. Sadi, R. W. Kelsall, and N. J. Pilgrim, "Investigation of SelfHeating Effects in Submicrometer GaN/GaN HEMTs Using an Electrothermal Monte Carlo Method," IEEE Trans. Electron Devices, vol. 53, pp. 2892-2900, December 2006

[4] F. Bonani, and G. Ghione, "On the Application of the Kirchhoff Transformation to the Steady-State Thermal Analysis of Semiconductor Devices with Temperature-Dependent and Piecewise Inhomogeneous Thermal conductivity," Solid-State Electronics, vol. 38, pp. 1409-1412, September 1995.

[5] J. Mateos, T. González, D. Pardo, V. Hoël, and A. Cappy, "Monte Carlo simulator for the design optimization of low-noise HEMTs," IEEE Trans. Electron Devices, vol 47, pp. 1950-1956, October 2000.

[6] S. García, S. Pérez, I. Íñiguez-de-la-Torre, J. Mateos, and T. González, "Comparative Monte Carlo analysis of InP- and GaNbased Gunn diodes," J. Appl. Phys, vol 115, 044510 (7 pp.) January 2014.

[7] S. García, I. Íñiguez-de-la-Torre, O. García-Pérez, J. Mateos, T. González, P. Sangaré, C. Gaquière and S. Pérez, "Self-consistent electro-thermal simulations of $\mathrm{AlGaN} / \mathrm{GaN}$ diodes by means of Monte Carlo method," Semicond. Sci. Technol, vol. 30, 035001 (8 pp.) January 2015.

[8] W. L. Liu and A. A. Balandin, "Temperature dependence of thermal conductivity of $\mathrm{Al}_{\mathrm{x}} \mathrm{Ga}_{1-\mathrm{x}} \mathrm{N}$ thin films measured by the differential $3 \omega$ technique," Appl. Phys. Lett, vol. 85, pp. 5230-5232, November 2004.

[9] S. Vitanov, V. Palankovski, S. Maroldt and R. Quay, "Hightemperature modeling of AlgaN/GaN HEMTs," Solid-State Electronics, vol. 54, pp. 1105-1112, June 2010. 